Artículo científico

Volumen 32(1):93-106. Enero-abril, 2021

e-ISSN 2215-3608, doi:10.15517/am.v32i1.42069

https://www.revistas.ucr.ac.cr/index.php/agromeso

\title{
Selección temprana en ensayos clonales de melina (Gmelina arborea Robx.) en Costa Rica ${ }^{1}$
}

\section{Early selection in clonal trials of melina (Gmelina arborea Robx.) in Costa Rica}

\author{
William Hernández-Castro ${ }^{2}$,Olman Murillo-Gamboa ${ }^{3}$,Yorleny Badilla-Valverde ${ }^{3}$
}

${ }^{1}$ Recepción: 1 de junio, 2020. Aceptación: 5 de octubre, 2020. Este trabajo formó parte de la tesis de doctorado del Doctorado en Ciencias Naturales para el Desarrollo (DOCINADE) del primer autor, el cual fue financiado parcialmente por el Consejo Nacional para Investigaciones Científicas y Tecnológicas (CONICIT).

2 Doctorado en Ciencias Naturales para el Desarrollo (DOCINADE), Universidad Nacional, Instituto Tecnológico de Costa Rica, Universidad Estatal a Distancia. Instituto de Investigación y Servicios Forestales de la Universidad Nacional de Costa Rica. Costa Rica. william.hernandez.castro@una.cr (autor para la correspondencia; https://orcid.org/0000-0003-2416-8329).

3 Instituto Tecnológico de Costa Rica, Escuela de Ingeniería Forestal, Costa Rica. omurillo@tec.ac.cr (https://orcid.org/0000-0003-32138867), y badilla@tec.ac.cr (https://orcid.org/0000-0002-6743-9734).

\section{Resumen}

Introducción. La edad de selección temprana permite acelerar los programas de mejoramiento genético y optimizar la producción forestal, en este caso con melina (Gmelina arborea), la cual es la segunda especie forestal más importante para la reforestación comercial en Costa Rica. Objetivo. Determinar la edad óptima de selección de melina en Costa Rica, mediante la evaluación de cuatro caracteres genéticos. Materiales y métodos. El estudio se llevó a cabo en Siquirres y Pérez Zeledón, región Caribe y zona sur de Costa Rica, respectivamente. Se utilizó un diseño de bloques completos al azar con cuatro repeticiones (sitios 1 y 3 ) y seis repeticiones (sitio 2) por tratamiento. El distanciamiento inicial fue de $4 \mathrm{~m}$ x $3 \mathrm{~m}$ para el ensayo del El Porvenir y de $4 \mathrm{~m}$ x $4 \mathrm{~m}$ para los ensayos de Siquirres y La Ceniza. Se calcularon parámetros genéticos a diferentes edades, para las variables diámetro a la altura del pecho (DAP), altura comercial, volumen comercial y calidad del fuste, con el software SELEGEN, el cual se basa en el método REML/BLUP, además, se realizaron correlaciones genéticas entre las variables evaluadas. Resultados. El DAP y el volumen comercial fueron los caracteres con mayor control genético, presentaron los mayores valores promedio de heredabilidad individual y media clonal, entre los 2,8 y 4 años de edad. Las mayores correlaciones genéticas (>80\%), entre las variables DAP y volumen comercial, se presentaron a partir de los 2,8 años de edad, que aumentan en edades posteriores hasta alcanzar valores mayores a $90 \%$. Conclusión. Pudo determinarse el rango entre los 2,8 y 4 años, como posible edad de selección genética para melina.

Palabras clave: heredabilidad, mejoramiento genético, parámetros genéticos, correlaciones genéticas.

\begin{abstract}
Introduction. The early selection age allows to accelerate genetic improvement programs and optimize forest production, in this case with melina (Gmelina arborea), which is the second most important forest species for
\end{abstract}


commercial reforestation in Costa Rica. Objective. To determine the optimal selection age of melina in Costa Rica, by evaluating four genetic characters. Materials and methods. The study was carried out in Siquirres and Pérez Zeledón, the Caribbean region and the southern zone of Costa Rica, respectively. A randomized complete block design was used with four replicates (Sites 1 and 3) and six replicates (Site 2) per treatment. The initial spacing was $4 \mathrm{~m} \mathrm{x} 3 \mathrm{~m}$ for the El Porvenir trial and $4 \mathrm{~m} \mathrm{x} 4 \mathrm{~m}$ for the Siquirres and La Ceniza trials. Genetic parameters were calculated at different ages, for the variables diameter at breast height (DBH), commercial height, commercial volume, and shaft quality, with the SELEGEN software, which is based on the REML / BLUP method, in addition, genetic correlations were made between the evaluated variables. Results. The DBH and the commercial volume were the characters with the greatest genetic control, they presented the highest mean values of individual heritability and clonal mean, between 2.8 and 4 years of age. The highest genetic correlations (> 80\%), between the DAP and commercial volume variables, occurred from 2.8 years of age, which increased in later ages until reaching values greater than $90 \%$. Conclusion. The range between 2.8 and 4 years could be determined as a possible age of genetic selection for melina.

Keywords: heritability, genetic improvement, genetic parameters, genetic correlations.

\section{Introducción}

La melina (Gmelina arborea) es una de las principales especies forestales utilizadas en proyectos de reforestación en Costa Rica, debido a las características de la madera y su versatilidad y fácil manejo, se ha posicionado en el mercado como la segunda especie de mayor demanda nacional. Actualmente, la madera de melina es utilizada para la fabricación de material de embalaje, para la industria de construcción y para la ebanistería en la fabricación de muebles (Moya, 2004; Barrantes \& Ugalde, 2019). La evolución que ha experimentado el sector forestal en Costa Rica en las últimas décadas y la aplicación de herramientas como la silvicultura clonal y la selección genética, han potenciado un aumento en la productividad de las plantaciones de Gmelina arborea.

El ciclo productivo de la melina es relativamente largo, el cual varía entre los cinco y ocho años, con base en el objetivo de producción, que comparado con otros usos de la tierra puede resultar poco competitivo, por lo tanto, es fundamental reducir el tiempo para obtener ganancias genéticas por ciclo de mejoramiento y aumentar la precisión en la selección genética para las características deseadas. La estimación de parámetros genéticos es de gran importancia en la planificación de los programas de mejoramiento genético, que direccionan los procedimientos de selección de individuos con características de interés para la producción forestal (Kumar, 2007; Zimback et al., 2011).

En las últimas décadas, en Costa Rica se han consolidado programas de mejoramiento genético, que han permitido acortar los turnos de corta y mejorar características de interés económico para el sector forestal. La experiencia del mejoramiento genético con melina se debe a que es fácil de reproducir, presenta rápido crecimiento y floración temprana (cortas generaciones de reproducción) y posee buena respuesta a la selección, además de presentar gran variación fenotípica combinada con una alta heredabilidad para caracteres importantes (Lauridsen \& Kjaer, 2002).

La edad de selección es importante en un programa de mejoramiento genético, si se considera que el tiempo es un factor determinante en la toma de decisiones, sobre todo en la actividad forestal, donde los resultados son a largo plazo y el desarrollo de estos programas es materia compleja, que requieren ser lo más eficientes posible. Para cualquier genetista forestal es clara la importancia de optimizar el tiempo demandado para realizar un ciclo de selección y, consecuentemente, promover la maximización de la ganancia genética por unidad de tiempo (Beltrame et al., 2012). 
La selección temprana es una estrategia a la cual recurre el genetista forestal para realizar selección de los mejores árboles, basado en alguna característica de interés económico, por lo general, el volumen comercial, aunque dicha selección se realice a una edad temprana, lo que se busca es mejorar la producción de volumen a la edad de rotación. Esta selección se realiza mucho antes de la edad de rotación para acortar el ciclo de reproducción y maximizar la ganancia por unidad de tiempo (Ye \& Jayawickrama, 2012). La mayor eficiencia en el proceso de selección incrementa las ganancias genéticas en un menor tiempo y disminuye los costos de los programas de mejoramiento (Moraes et al., 2014).

La eficiencia de un programa de reproducción forestal está directamente relacionada con el tiempo requerido para obtener ganancias genéticas. Por lo tanto, la coincidencia del desempeño de las características de interés, en edades tempranas y adultas, es esencial para una mayor efectividad de un programa de mejoramiento genético (Santos et al., 2014). La variación entre clones se usa comúnmente como una estimación de la variación genética total y para calcular el grado de control genético para un carácter particular (Foster \& Shaw, 1988).

Para evaluar adecuadamente la efectividad de la selección temprana y determinar con precisión la edad de selección óptima, se requiere de una comprensión completa de los parámetros genéticos. Dentro de los más relevantes se encuentran los coeficientes de heredabilidades y de las correlaciones genéticas edad-edad, de los distintos caracteres a diferentes edades (Chen et al., 2003; Diao et al., 2016). Estudios relacionados con la estimación de parámetros genéticos realizados en un ensayo de polinización abierta con melina en Malasia, mostraron resultados de heredabilidad individual para la variable DAP de $h^{2} g=0,12$ a la edad de 5 y 6 años y en altura comercial de $h^{2} g=0,068, h^{2} g=0,179$ y $h^{2} g=0,166$ a la edad de 1,5 y 6 años, respectivamente (Lokmal, 1994), por otra parte, Hodge \& Dvorak (2004), obtuvieron valores de heredabilidad para el DAP de $h^{2} g=0,10$ y $h^{2} g=0,09$, a la edad de 1 y 3 años, respectivamente, y en altura de $h^{2} g=0,18$ y $h^{2} g=0,17$ a la edad de 5 y 6 años, respectivamente. Otros resultados reportados para melina, en la India, en un estudio con setenta clones, señala heredabilidades en sentido amplio para la altura de $h^{2} g=0,31$ a los 5 años de edad (Kumar, 2007).

La selección temprana tiene la ventaja que permite el establecimiento de ensayos de campo más cortos en el tiempo, mayor facilidad para la recolección de datos y mayor flexibilidad con relación a los cambios de los objetivos propuestos para el programa de mejoramiento (Dean et al., 2006). Lo anterior constituye la principal limitación a la hora de realizar investigaciones como la presente, en vista de que es difícil contar con ensayos establecidos de manera paralela y condiciones de sitio diferentes que permitan conocer las respuestas del material genético utilizado. Por lo tanto, el presente estudio tuvo como objetivo determinar la edad óptima de selección de melina en Costa Rica, mediante la evaluación de cuatro caracteres genéticos.

\section{Materiales y métodos}

Se establecieron tres ensayos de melina (Gmelina arborea), uno ubicado en Siquirres, provincia de Limón, Costa Rica, denominado ensayo Siquirres o sitio 1, los otros corresponden a dos ensayos localizados en Pérez Zeledón, San José, Costa Rica, denominados ensayo La Ceniza (sitio 2) y ensayo El Porvenir (sitio 3), con la finalidad de obtener la información para los análisis genéticos de los clones y material testigo utilizados (Cuadro 1).

El sitio 1 se presenta con una topografía casi plana con pendientes inferiores a $5 \%$, el tipo de suelo se clasifica dentro del orden Inceptisol (Mata-Chinchilla \& Castro-Chinchilla, 2019). El área de estudio se ubica dentro de la zona de vida Bosque muy Húmedo Tropical, con una altitud de $97 \mathrm{~m}$, presenta precipitaciones durante todo el año, con una media anual de $3630 \mathrm{~mm}$ al año. La temperatura media anual es de $25,9^{\circ} \mathrm{C}$ (Instituto de Desarrollo Rural, 2015). Los sitios 2 y 3, son terrenos de uso agrícola, con pendiente plana a ondulada, presentan suelos del orden Ultisol (Mata-Chinchilla \& Castro Chinchilla, 2019). La temperatura promedio anual oscila entre los $24-26^{\circ} \mathrm{C}$, la precipitación media anual es de 3000-4000 mm (Instituto Meteorológico Nacional, 2005), y una humedad relativa 
Cuadro 1. Fecha de establecimiento, material genético utilizado y localización geográfica de ensayos genéticos de melina (Gmelina arborea), zona atlántica y zona sur de Costa Rica.

Table 1. Date of establishment, genetic material used, and geographical location of genetic tests of melina (Gmelina arborea), Atlantic zone and south zone of Costa Rica.

\begin{tabular}{|c|c|c|c|c|c|}
\hline Sitio & Ensayo genético & $\begin{array}{c}\text { Fecha de } \\
\text { establecimiento }\end{array}$ & $\begin{array}{l}\text { Material genético } \\
\qquad *\end{array}$ & Latitud & Longitud \\
\hline Sitio 1 & Siquirres & Junio 2010 & $\begin{array}{l}25 \text { clones } \\
2 \text { testigos }\end{array}$ & N 10¹0'80,9'” & W 835' $9,7^{\prime}$ \\
\hline Sitio 2 & La Ceniza & Octubre 2013 & $\begin{array}{l}29 \text { clones } \\
5 \text { testigos }\end{array}$ & N 9॰30’16,0”' & W $83^{\circ} 68^{\prime} 23,9^{\prime \prime}$ \\
\hline Sitio 3 & El Porvenir & Octubre 2010 & $\begin{array}{l}36 \text { clones } \\
1 \text { testigos }\end{array}$ & N 9²4'70,0”' & W $83^{\circ} 54^{\prime} 83,8^{\prime \prime}$ \\
\hline
\end{tabular}

*Clones pertenecientes a CoopeAgri R.L., ASIREA y GENFORES y el material testigo proviene de rodal semillero del Centro Agronómico Tropical de Investigación y Enseñanza (CATIE) y del Banco de semillas del CATIE / * Clones belonging to CoopeAgri R.L., ASIREA, and GENFORES and the control material comes from Centro Agronómico Tropical de Investigación y Enseñanza (CATIE) seed stand and CATIE Seed Bank.

promedio de $80 \%$, la región se localiza en la zona de vida Bosque muy Húmedo Premontano, con una altitud promedio de $700 \mathrm{msnm}$ (Alfaro et al., 2013).

En el sitio 1 los clones utilizados en el ensayo de Siquirres fueron los que comercializa la Asociación para el Desarrollo Sostenible de la Región Atlántica (ASIREA). Uno de los testigos correspondió a plantas provenientes de semilla del Banco de Semillas del Centro Agronómico Tropical de Investigación y Enseñanza (CATIE) y el otro testigo provino de semilla de rodal semillero de Hojancha, Guanacaste.

Para el caso del ensayo de La Ceniza en el sitio 2, los clones pertenecían a la Cooperativa CoopeAgri R. L., los cuales son utilizados para la reforestación comercial en la zona sur de Costa Rica, el testigo 1 provino del Centro Agrícola Cantonal de Hojancha $\left(\mathrm{CACH}, \mathrm{T}_{1}\right)$, el testigo 2 provino de Ston Forestal $\left(\right.$ Ston, $\left.\mathrm{T}_{2}\right)$, clones de la empresa Brinkman y Asociados Reforestadores de Centro América S.A. (BARCA, $\mathrm{T}_{3}$ ), Instituto de Investigación y Servicios Forestales (INISEFOR, $\mathrm{T}_{4}$ ), y un último testigo proveniente del material genético utilizado en la zona norte $\left(\mathrm{T}_{5}\right)$ de Costa Rica.

Los clones del ensayo El Porvenir (sitio 3) provinieron de la Cooperativa Genética Forestal, la cual agremia varias empresas que se dedican a la reforestación comercial con melina, el testigo utilizado provino de rodal semillero del CATIE ubicado en El Ceibo de Buenos Aires, provincia de Puntarenas.

\section{Diseño experimental}

Para los tres ensayos se utilizó un diseño experimental de bloques completos al azar (seis bloques), con cuatro repeticiones, a excepción del sitio 2: La Ceniza, donde se utilizaron seis repeticiones por tratamiento, debido a que se disponía de un área mayor. Los clones fueron distribuidos aleatoriamente en parejas dentro de cada bloque, con la condición de que no quedaran juntas, el mismo tratamiento (parejas) dentro de un mismo bloque.

La densidad inicial para el sitio 1 y 2 fue de 625 árboles por hectárea (distanciamiento de $4 \mathrm{~m} \mathrm{x} 4 \mathrm{~m}$ ), mientras que para el sitio 3: El Porvenir se estableció utilizando una densidad de 833 árboles por hectárea, con un distanciamiento de $4 \mathrm{~m} \mathrm{x} 3 \mathrm{~m}$, la diferencia en el distanciamiento entre sitios tuvo relación con el tipo de manejo adoptado por cada propietario, ya que para la época, la recomendación de sembrar a una densidad mayor o menor a las establecidas en los sitios bajo estudio no estaban muy definidas a nivel país. Con relación a la preparación del suelo, para el caso del sitio 1 se realizó una limpieza previa del terreno que brindó las condiciones para el establecimiento de la 
plantación, posteriormente, se realizaron limpiezas de mantenimiento. En el caso de los dos ensayos (sitio 2 y sitio 3), localizados en Pérez Zeledón, la preparación del suelo consistió en una limpieza previa, quitando la vegetación existente por medio de chapeas y posterior limpieza en el punto donde se plantó cada árbol (rodaja).

\section{Recolección y análisis de la información}

Los tres ensayos fueron evaluados a diferentes edades, debido a que se plantaron en distintas fechas, estandarizando las evaluaciones de manera parcial en los tres sitios. El ensayo de Siquirres (sitio 1) fue medido a la edad de 1,5 años, 2,8 años, 4 años, 4,9 años y 5,9 años; para el caso del ensayo La Ceniza (sitio 2), se midió a los 1,5 años, 2,7 años, 3,5 años y 4,5 años, mientras que el ensayo El Porvenir (sitio 3) se contó con información de mediciones fiables a la edad de 4,5 años y 5,8 años. Para el caso del sitio 3, la información sobre mediciones antes de los 4 años fue incompleta, lo que limitó realizar correlaciones genéticas y la estimación de parámetros genéticos para las variables evaluadas. Se incorporaron las mediciones a los 4,5 y 5,8 , con la finalidad de contar con más información en el analices de las variables estudiadas a edades superiores a los 4 años, también por el número importante de clones que conformaron dicho ensayo.

Los resultados para los caracteres cuantitativos se analizaron tomando en cuenta los valores de los parámetros genéticos de los tres ensayos en conjunto (sitio 1, sitio 2 y sitio 3). Se analizó la información con base en los siguientes rangos de edad; menor de dos años $(<2)$, entre dos y tres años $(2-3)$, entre tres y cuatro años $(3-4)$ y mayor a cuatro años $(>4)$. El análisis integrado de los tres ensayos se realizó con base en el comportamiento individual, el cual mostró una tendencia similar al comportamiento obtenido al realizar el análisis de toda la información en su conjunto. Es claro que los coeficientes de los parámetros genéticos variaron entre un ensayo y otro, sin embargo, la tendencia se mantuvo al integrarlos de manera conjunta.

Se utilizaron hojas de campo y el respectivo croquis que mostraba el diseño de cada ensayo. Se midieron las variables diámetro a la altura de pecho (DAP), altura comercial y la calidad del fuste comercial, que se cuantifica con base en la calidad de las primeras cuatro trozas, con una longitud de 2,5 m cada una, donde 1 es la máxima calidad para aserrío y 4 es una troza sin valor comercial o utilizable para leña.

\section{Análisis de datos}

Para el análisis de la información se procedió a elaborar una base de datos que integró las diferentes mediciones de las variables evaluadas a cada clon y para cada uno de los ensayos. Posteriormente, a partir de los datos de DAP y altura comercial, se procedió a calcular el volumen comercial utilizando la siguiente ecuación (1).

$$
\mathrm{Vc}=\left((\mathrm{D} / 100)^{2 *}(\pi / 4) *(\mathrm{Hcom})\right) * 0,65
$$

Donde

Vc: volumen comercial.

D: diámetro a la altura del pecho en $\mathrm{cm}$.

Hcom: altura comercial estimada a partir de la cantidad de trozas comerciales posibles de 2,5 m largo en cada árbol.

Para el cálculo de la calidad del fuste se tomó en cuenta la cantidad de trozas estimadas por individuo y el valor asignado según la calificación para cada troza, para esto se utilizó la metodología para la valoración de plantaciones forestales desarrollada por Murillo et al. (2004). La ecuación 2 permitió calcular la calidad global del árbol, tomando en cuenta el aporte individual de cada troza; en ese caso se corresponde a un árbol cuyo fuste cuenta con cuatro trozas. 
Calidad general $=\mathrm{T} 1 * 0,40+\mathrm{T} 2 * 0,30+\mathrm{T} 3 * 0,20+\mathrm{T} 4 * 0,10$

Donde: $T 1, T 2, T 3$ y $T 4$ : calidades (en escala de 1 a 4 ) asignadas a cada toza.

En vista de que la calidad general del árbol osciló entre 1 y 4, se procedió a transformar la calidad en una escala de 1 a 100, para lo cual se utilizó el algoritmo descrito por la ecuación 3.

Calidad $(\%)=100 *[1-(($ calidad general-1)/3) $]$

Posteriormente, se procedió a conformar una base de datos en Excel, que unificara para cada clon dentro de cada bloque todas las variables evaluadas, para cada una de las mediciones realizadas para su respectivo procesamiento. Una vez obtenidos los resultados de los ensayos, se procedió a graficar las heredabilidades (individual y media clonal), para obtener un modelo de selección temprana, que reflejara el comportamiento de los coeficientes de heredabilidad y establecer a partir del mismo una posible edad de selección, fundamentado, en un análisis de correlaciones genéticas edad-edad, entre los caracteres evaluados.

El análisis de los datos de los clones se realizó con base en el modelo 2 de SELEGEN, específico para ensayos clonales no emparentados, en una sola localidad, varias plantas por parcela:

$y=X r+Z g+W p+e$

Donde,

$y$ : es el vector de datos.

$r$ : es el vector del efecto de repetición (asumido como fijo) y sumados a la media general.

$g$ : es el vector del efecto genético total (asumido como aleatorio).

$p$ : es el vector de los efectos de parcela.

$e$ : es el vector del término del error o residuo (aleatorio).

Las letras mayúsculas representan las matrices de incidencia para los efectos referidos (de Resende, 2016). Posteriormente, se realizaron correlaciones genéticas que permitieron analizar interrelaciones entre las diversas variables evaluadas, a través del modelo estadístico 102 del software SELEGEN (de Resende, 2016).

\section{Resultados}

Los mayores valores de los coeficientes de heredabilidad individual en sentido amplio y heredabilidad media del clon para los caracteres evaluados se presentaron para el diámetro a la altura de pecho (DAP) y el volumen comercial, y correspondieron a los ensayos de La Ceniza y Siquirres (sitio 1 y sitio 2). Sin embargo, no existió un comportamiento definido en este carácter y se observó que los valores de heredabilidad variaron entre los sitios evaluados y entre los rangos de edad establecidos. Si se analiza a nivel de sitio, de manera independiente a los demás, la heredabilidad individual y media clonal máxima, para los caracteres DAP y altura comercial evaluados en el ensayo de Siquirres (sitio 1), se presentaron en el rango de 3-4 años, mientras que para el volumen comercial los valores máximos de heredabilidad se presentaron posterior a la edad de cuatro años. Con relación al ensayo de La Ceniza (sitio 2) los valores máximos se expresaron a partir de los cuatro años de edad para las variables evaluadas, tanto para la heredabilidad individual en sentido amplio, como para la heredabilidad media del clon (Cuadro 2). El sitio 3 (El Porvenir), evidentemente los valores de las heredabilidades tuvieron su aporte en el último rango de ISSN 2215-3608 doi:10.15517/am.v32i1.42069 
Cuadro 2. Heredabilidad individual en sentido amplio y heredabilidad media del clon para las variables DAP, volumen comercial y altura comercial de melina (Gmelina arborea), en los sitios: Siquirres y Pérez Zeledón, Costa Rica. 2013-2018.

Table 2. Individual heritability in the broad sense and mean heritability of the clone for the variables DAP, commercial volume, and commercial height of melina (Gmelina arborea), in the sites: Siquirres and Pérez Zeledón, Costa Rica. 2013-2018.

\section{Diámetro a la altura del pecho (DAP)}

\begin{tabular}{|c|c|c|c|c|c|c|c|c|}
\hline \multirow[b]{2}{*}{ Edad (años) } & \multicolumn{4}{|c|}{ Heredabilidad individual } & \multicolumn{4}{|c|}{ Heredabilidad media del clon } \\
\hline & Sitio 1 & Sitio 2 & Sitio 3 & Promedio & Sitio 1 & Sitio 2 & Sitio 3 & Promedio \\
\hline$<2$ & 0,096 & 0,040 & & 0,068 & 0,685 & 0,557 & & 0,621 \\
\hline$(2-3)$ & 0,087 & 0,102 & & 0,094 & 0,690 & 0,760 & & 0,725 \\
\hline$(3-4)$ & 0,127 & 0,147 & & 0,137 & 0,718 & 0,828 & & 0,773 \\
\hline \multirow[t]{3}{*}{$>4$} & 0,105 & 0,153 & 0,080 & 0,113 & 0,536 & 0,849 & 0,658 & 0,681 \\
\hline & \multicolumn{8}{|c|}{ Volumen comercial } \\
\hline & \multicolumn{4}{|c|}{ Heredabilidad individual } & \multicolumn{4}{|c|}{ Heredabilidad media del clon } \\
\hline Edad (años) & Sitio 1 & Sitio 2 & Sitio 3 & Promedio & Sitio 1 & Sitio 2 & Sitio 3 & Promedio \\
\hline$<2$ & 0,090 & 0,012 & & 0,051 & 0,691 & 0,269 & & 0,480 \\
\hline$(2-3)$ & 0,079 & 0,063 & & 0,071 & 0,669 & 0,652 & & 0,660 \\
\hline$(3-4)$ & 0,111 & 0,092 & & 0,102 & 0,689 & 0,749 & & 0,719 \\
\hline \multirow[t]{3}{*}{$>4$} & 0,114 & 0,113 & 0,064 & 0,097 & 0,594 & 0,812 & 0,550 & 0,652 \\
\hline & \multicolumn{8}{|c|}{ Altura comercial } \\
\hline & \multicolumn{4}{|c|}{ Heredabilidad individual } & \multicolumn{4}{|c|}{ Heredabilidad media del clon } \\
\hline Edad (años) & Sitio 1 & Sitio 2 & Sitio 3 & Promedio & Sitio 1 & Sitio 2 & Sitio 3 & Promedio \\
\hline$<2$ & 0,035 & 0,0624 & & 0,049 & 0,465 & 0,626 & & 0,545 \\
\hline$(2-3)$ & 0,034 & 0,035 & & 0,035 & 0,461 & 0,505 & & 0,483 \\
\hline$(3-4)$ & 0,061 & 0,0455 & & 0,053 & 0,533 & 0,552 & & 0,542 \\
\hline \multirow[t]{3}{*}{$>4$} & 0,052 & 0,0749 & 0,013 & 0,047 & 0,366 & 0,706 & 0,223 & 0,432 \\
\hline & \multicolumn{8}{|c|}{ Calidad del fuste } \\
\hline & \multicolumn{4}{|c|}{ Heredabilidad individual } & \multicolumn{4}{|c|}{ Heredabilidad media del clon } \\
\hline Edad (años) & Sitio 1 & Sitio 2 & Sitio 3 & Promedio & Sitio 1 & Sitio 2 & Sitio 3 & Promedio \\
\hline$<2$ & 0,083 & 0,011 & & 0,047 & 0,664 & & & 0,664 \\
\hline$(2-3)$ & 0,079 & 0,015 & & 0,047 & 0,650 & 0,123 & & 0,094 \\
\hline$(3-4)$ & 0,102 & 0,047 & & 0,074 & 0,657 & 0,141 & & 0,399 \\
\hline$>4$ & 0,125 & & 0,073 & 0,099 & 0,623 & 0,627 & 0,698 & 0,662 \\
\hline
\end{tabular}

* El sitio 1 corresponde al ensayo establecido en Siquirres y los sitios 2 y 3, corresponden a los ensayos La Ceniza y El Porvenir, respectivamente /* Site 1 corresponds to the test established in Siquirres and sites 2 and 3 correspond to the La Ceniza and El Porvenir trials, respectively.

edad, sin embargo, lo importante en este caso era conocer cuál variable resultó tener los mayores coeficientes de heredabilidad.

Al integrar el análisis tomando en cuenta los coeficientes de los parámetros genéticos de los caracteres cuantitativos evaluados, los mayores valores promedio por rango de edad correspondieron al DAP, al volumen comercial y a la altura comercial, con una heredabilidad en sentido amplio de $0,137,0,102$ y 0,053 , respectivamente: por otra parte, para la heredabilidad media del clon, los valores más altos correspondieron a $0,773,0,719$ y 0,542 , respectivamente, para ese mismo rango de edad (Cuadro 2). 
La calidad del fuste, por ser una variable cualitativa, se comportó de manera diferente al resto, donde los mayores coeficientes de las heredabilidades se presentaron en el ensayo de Siquirres (sitio 1). Al analizar los resultados de manera conjunta, tomando en cuenta los tres ensayos, los valores tendieron a disminuir significativamente, en este caso el sitio 2. La Ceniza, se caracterizó por presentar un alto grado de bifurcación que influyó sobre los valores promedio de los coeficientes de heredabilidad individual y media del clon (Cuadro 2). Se observó que los mayores valores de heredabilidad se registraron para la variable DAP, esto para los diferentes rangos de edad, seguido por el volumen comercial y la altura comercial.

El comportamiento de la heredabilidad individual en sentido amplio y heredabilidad media clonal fue ascendente a partir del año 1 y hasta el año 4, para las variables DAP, volumen comercial, altura comercial y calidad del fuste (Figura 1). Los mayores coeficientes de determinación de las ecuaciones de mejor ajuste de los datos se presentaron con los valores de heredabilidad media del clon, sin embargo, el comportamiento fue similar para ambas heredabilidades, que mostraron los valores más altos para la variable DAP, con un valor de $\mathrm{R}^{2}=0,88$ y $\mathrm{R}^{2}=0,85$, para heredabilidad individual y media del clon, respectivamente (Figura 1).

a)

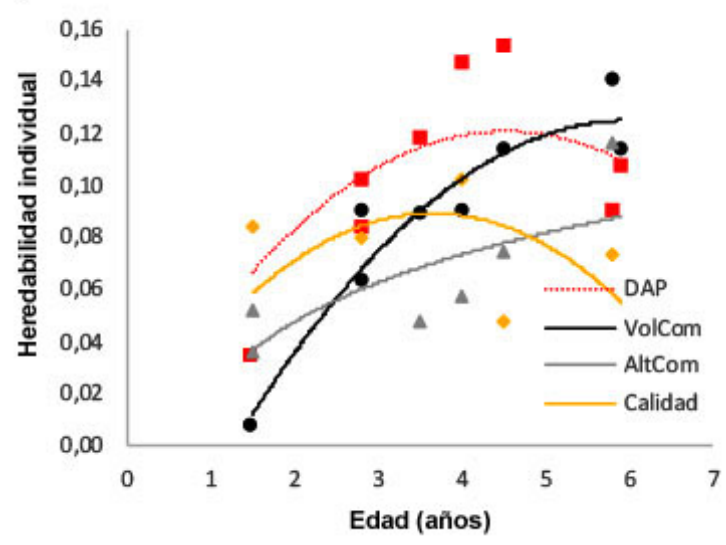

$\mathrm{DAP}=-0,0128 \mathrm{x}^{2}+0,1104 \mathrm{x}-0,1093$

VolCom $=-0,0055 x^{2}+0,0665 x-0,0754$

AltCom $=0,0063 x^{2}-0,0293 x+0,0727$

Calidad $=-0,0033 \mathrm{x}^{2}+0,0232 \mathrm{x}+0,0516$

$$
\begin{aligned}
& \mathrm{R}^{2}=0,88 \\
& \mathrm{R}^{2}=0,90 \\
& \mathrm{R}^{2}=0,96 \\
& \mathrm{R}^{2}=0,44
\end{aligned}
$$

b)

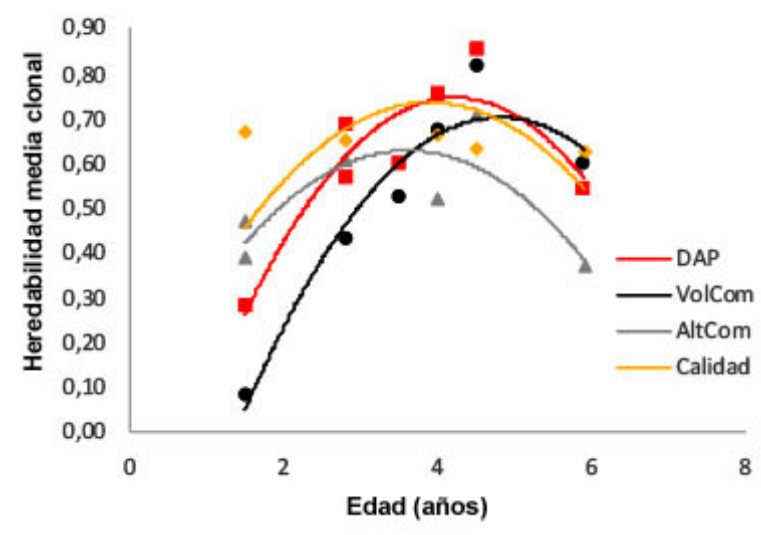

$$
\begin{array}{ll}
\text { DAP }=-0,0557 \times 2+0,4643 x-0,2743 & R^{2}=0,85 \\
\text { VolCom }=-0,052 \times 2+0,5047 x-0,5679 & R^{2}=0,97 \\
\text { AltCom }=-0,0353 \times 2+0,2439 x+0,1469 & R^{2}=0,80 \\
\text { Calidad }=-0,002 \times 2+0,0065 x+0,6559 & R^{2}=0,86
\end{array}
$$

Figura 1. a) Comportamiento de la heredabilidad individual y en b) heredabilidad media clonal, en función de la edad para las variables diámetro a la altura del pecho (DAP), volumen comercial (VolCom), altura comercial (AltCom), y calidad del fuste, en melina (Gmelina arborea), en Siquirres (sitio 1) y Pérez Zeledón (sitio 2 y 3), Costa Rica. 2013-2018.

Figure 1. a) Behavior of individual heritability and in b) mean clonal heritability, as a function of age for the variables diameter at breast height (DAP), commercial volume (VolCom), commercial height (AltCom), and stem quality in melina (Gmelina arborea), in Siquirres (site 1) and Pérez Zeledón (site 2 y 3), Costa Rica. 2013-2018.

En el caso de la heredabilidad individual los caracteres volumen comercial y altura comercial presentaron un valor de $\mathrm{R}^{2}=0,90$ y 0,96 , respectivamente, mientras que la calidad del fuste reflejó un valor de $\mathrm{R}^{2}=0,44$, esta variable cualitativa se caracterizó porque su comportamiento tuvo relación con el manejo silvicultural de la plantación.

Los mayores valores para el coeficiente de determinación para la heredabilidad media del clon, varía con relación a los valores de heredabilidad individual, en este caso, el volumen comercial reflejó el valor máximo, seguido de la calidad, DAP y la altura comercial, con valores de $\mathrm{R}^{2}=0,97,0,86,0,85$ y 0,80 , respectivamente (Figura 1 ). 
En el ensayo de Siquirres las mayores correlaciones se presentaron entre las variables DAP y volumen comercial con un valor máximo de correlación de 0,98, a la edad de 2,8 años. Esto representó una posible proyección de edad de selección, ya que la correlación edad-edad permitió conocer que tan estable se mantuvo el ranking genético de los conjuntos genéticos evaluados. La tendencia fue un aumento en los valores de correlación entre estas dos variables conforme aumentó la edad, sin embargo, a la edad de 5,9 años, se reflejó una disminución con un valor de correlación de 0,85 (Cuadro 3).

Cuadro 3. Correlación genética entre las variables diámetro a la altura del pecho (DAP), altura comercial (AltCom) y volumen comercial (VolCom) de ensayo de melina (Gmelina arborea), ubicado en sitio 1: Siquirres, Costa Rica. 2013-2018.

Table 3. Genetic correlation between the variables diameter at breast height (DAP), commercial height (AltCom), and commercial volume (VolCom) of the melina (Gmelina arborea) test located in Siquirres, Costa Rica. 2013-2018.

\begin{tabular}{|c|c|c|c|c|c|c|c|c|c|c|c|}
\hline \multirow{3}{*}{$\begin{array}{l}\begin{array}{l}\text { Edad } \\
\text { (años) }\end{array} \\
\text { Variables }\end{array}$} & \multicolumn{11}{|c|}{ Ensayo Siquirres } \\
\hline & \multicolumn{2}{|c|}{1,5} & \multicolumn{3}{|c|}{2,8} & \multicolumn{3}{|c|}{4} & \multicolumn{3}{|c|}{5,9} \\
\hline & AltCom & VolCom & DAP & AltCom & VolCom & DAP & AltCom & VolCom & DAP & AltCom & VolCom \\
\hline DAP & 0,69 & 0,95 & 0,87 & 0,25 & 0,83 & 0,69 & 0,41 & 0,66 & 0,57 & 0,27 & 0,50 \\
\hline AltCom & & 0,83 & 0,80 & 0,65 & 0,79 & 0,54 & 0,26 & 0,50 & 0,37 & 0,19 & 0,34 \\
\hline VolCom & & & 0,90 & 0,39 & 0,89 & 0,67 & 0,42 & 0,66 & 0,53 & 0,28 & 0,49 \\
\hline DAP & & & & 0,44 & 0,98 & 0,79 & 0,49 & 0,78 & 0,65 & 0,38 & 0,62 \\
\hline AltCom & & & & & 0,50 & 0,24 & 0,26 & 0,30 & $-0,10$ & 0,08 & $-0,02$ \\
\hline VolCom & & & & & & 0,77 & 0,50 & 0,77 & 0,59 & 0,42 & 0,62 \\
\hline DAP & & & & & & & 0,59 & 0,94 & 0,81 & 0,43 & 0,74 \\
\hline AltCom & & & & & & & & 0,80 & 0,39 & 0,46 & 0,49 \\
\hline VolCom & & & & & & & & & 0,75 & 0,46 & 0,72 \\
\hline DAP & & & & & & & & & & 0,36 & 0,85 \\
\hline AltCom & & & & & & & & & & & 0,78 \\
\hline
\end{tabular}

Para el ensayo La Ceniza (sitio 2), las correlaciones más altas se presentaron a partir de los 2,7 años de edad, para todas las variables analizadas entre sí. El comportamiento fue similar al ensayo de Siquirres (sitio 1), donde el DAP y el volumen comercial a la edad de 2,7 años, presentaron una correlación de 0,95 . Sin embargo, se observó la tendencia de aumentar los valores de correlación conforme aumentó la edad, que para el caso del DAP y el volumen comercial fue bastante alta durante todo el período de evaluación.

Al analizar las correlaciones entre el DAP y la altura comercial el comportamiento fue similar al DAP y volumen comercial; se evidenció un aumento en los valores de correlación conforme aumentó la edad, con la diferencia de que fueron menores. Para el caso de las correlaciones genéticas entre la altura comercial y el volumen comercial, se presentaron valores mayores a partir de los 2,7 años de edad (Cuadro 4).

El análisis de correlación para el caso del ensayo El Porvenir (sitio 3), evidenció que la mayor correlación entre las variables DAP y volumen comercial fue a los 4,5 años de edad $(0,84)$; luego al correlacionar el DAP entre sí, a las edades de 4,5 y 5,8 años, se reflejó una correlación de 0,95 ; posteriormente, a la edad de 5,8 años se obtuvo una correlación de 0,83 entre las variables DAP y volumen comercial. 
Cuadro 4. Correlación genética entre las variables diámetro a la altura del pecho (DAP), altura comercial (AltCom) y volumen comercial (VolCom) de dos ensayos de melina (Gmelina arborea), ubicados en sitio 2: Pérez Zeledón, Costa Rica. $2013-2018$.

Table 4. Genetic correlation between the variables diameter at breast height (DAP), commercial height (AltCom), and commercial volume (VolCom) from two melina (Gmelina arborea) trials located in Site 2: Pérez Zeledón, Costa Rica. 2013-2018.

\begin{tabular}{|c|c|c|c|c|c|c|c|c|c|c|c|}
\hline \multirow{3}{*}{$\begin{array}{l}\text { Edad } \\
\text { (años) }\end{array}$} & \multicolumn{11}{|c|}{ Ensayo La Ceniza } \\
\hline & \multicolumn{2}{|c|}{1,5} & \multicolumn{3}{|c|}{2,7} & \multicolumn{3}{|c|}{3,4} & \multicolumn{3}{|c|}{4,5} \\
\hline & AltCom & VolCom & DAP & AltCom & VolCom & DAP & AltCom & VolCom & DAP & AltCom & VolCom \\
\hline DAP & 0,61 & 0,87 & 0,73 & 0,73 & 0,78 & 0,67 & 0,74 & 0,77 & 0,62 & 0,76 & 0,71 \\
\hline AltCom & & 0,52 & 0,19 & 0,46 & 0,25 & 0,08 & 0,48 & 0,19 & 0,02 & 0,43 & 0,10 \\
\hline VolCom & & & 0,51 & 0,58 & 0,62 & 0,49 & 0,60 & 0,66 & 0,45 & 0,56 & 0,57 \\
\hline DAP & & & & 0,76 & 0,95 & 0,95 & 0,68 & 0,93 & 0,92 & 0,82 & 0,91 \\
\hline AltCom & & & & & 0,85 & 0,65 & 0,81 & 0,75 & 0,63 & 0,81 & 0,69 \\
\hline VolCom & & & & & & 0,89 & 0,71 & 0,94 & 0,87 & 0,84 & 0,91 \\
\hline DAP & & & & & & & 0,65 & 0,94 & 0,97 & 0,78 & 0,95 \\
\hline AltCom & & & & & & & & 0,76 & 0,62 & 0,83 & 0,66 \\
\hline VolCom & & & & & & & & & 0,91 & 0,82 & 0,94 \\
\hline DAP & & & & & & & & & & 0,81 & 0,97 \\
\hline AltCom & & & & & & & & & & & 0,84 \\
\hline
\end{tabular}

\section{Discusión}

Los valores de heredabilidad individual en sentido amplio, para el carácter DAP en melina, se pueden considerar bajos de acuerdo con de Resende \& Duarte (2007), que señalaron que heredabilidades de 0,01 a 0,15 son bajas, de 0,16 a 0,50 son intermedias y superiores a 0,50 son altas.

Con relación a la heredabilidad media clonal, se presentó el mismo comportamiento que la heredabilidad individual, sin embargo, con valores más altos, lo que presupone es un mayor control genético en términos de la media a nivel de clones. La tendencia de la heredabilidad individual y media clonal, de acuerdo con los resultados obtenidos en los diferentes ensayos, evidenciaron como posible rango de edad de selección genética entre los 2,8 años y 4 años, período en el cual los valores de los estimados tendieron a estabilizarse. De acuerdo con Xiang et al. (2003), el DAP puede ser un mejor predictor para la selección temprana.

La calidad del fuste es una variable que está determinada en gran medida por la altura de bifurcación (Pavlotzky \& Murillo, 2014), situación que se reflejó en el sitio 2, donde los valores de heredabilidad individual y media clonal fueron muy bajos. La tendencia que mostraron los datos fue a aumentar los valores de los parámetros genéticos a partir de los 2,8 años hasta los 5,9 años de edad, para el caso específico de este carácter. La situación varió para cada uno de los ensayos, donde el sitio 1, Siquirres, fue el que presentó un comportamiento más estable de la heredabilidad individual y heredabilidad media clonal, dichas diferencias de comportamiento para la calidad del fuste, puede que estuvieran relacionadas con el manejo silvicultural que recibieron los ensayos, así como también por las diferencias de tipo edafoclimáticas, en el sitio 1 predominaban suelos Inceptisoles, mientras que los sitios 2 y 3 se presentaban suelos del orden ultisol, condición que aunado con el manejo y las condiciones climáticas, pudieron marcar diferencias en la respuesta del crecimiento de los árboles.

Los resultados en volumen comercial para los diferentes parámetros genéticos presentaron un comportamiento similar al DAP y en menor grado con la altura. Valores de heredabilidad individual en volumen comercial 
reportados en Tailandia, mostraron un valor de $h^{2} g=0,11$ para melina, a la edad de 2 y 3 años (Wijoyo, 2000), los cuales difirieron de los obtenidos en este estudio, sin embargo, analizando los valores de heredabilidad individual y media clonal obtenidos, las posibilidades de selección a partir del volumen comercial son factibles. Los resultados en términos de la heredabilidad individual en sentido amplio y heredabilidad media clonal, mostraron la tendencia que permite suponer como posible edad de selección entre los dos y cuatro años de edad, con base al volumen comercial (Figura 1a y 1b).

El comportamiento de los estimados de los parámetros genéticos obtenidos en este trabajo, tendieron a diferir entre los sitios evaluados, lo que evidenció no solo variación a nivel genético, sino también la posible influencia de factores edáficos y climáticos, los cuales no fueron objeto de análisis en este trabajo, en vista de que se deseaba conocer diferencias entre la variabilidad genética de los clones utilizados y el material testigo, tomando en cuenta las diferencias en el manejo silvicultural que recibieron los tres ensayos.

Estudios previos con melina, demostraron que la altura comercial pudo ser una variable que funcionó como predictora de la selección genética, sin embargo, dichos trabajos hicieron referencia a investigaciones con plantaciones forestales localizadas en regiones donde la melina es nativa (Padua, 2004), aspecto que pudo influir en los resultados, en vista de que a pesar de que la especie crece bien en el trópico, existen factores asociados que también influyen en el crecimiento de la especie, lo que puede explicar las diferencias en los valores de los coeficientes de heredabilidad obtenidos para la variable altura, sin embargo, la altura comercial como variable de selección, se evidenció mejor en términos de la tendencia de la heredabilidad media del clon, entre los 2 años y 4 años de edad donde se reflejaron los mayores valores para este carácter (Figura 1a y 1b).

Los valores de las correlaciones genéticas obtenidos en los tres ensayos para los caracteres diámetro a la altura del pecho (DAP), altura comercial (AltCom) y volumen comercial (VolCom), permitieron conocer el potencial de la selección temprana de los materiales genéticos utilizados. Las correlaciones entre el DAP y volumen comercial a diferentes edades permitieron obtener valores de correlación mayores a $73 \%$, a partir de los cuatro años de edad, aunque para el sitio 1 y 2 , los mayores valores de correlación entre los caracteres evaluados se presentaron a partir de la edad de 2,7 y 2,8 años, respectivamente, con valores altos de correlación para DAP y volumen comercial ( $>95$ $\%$ ), estos valores coincidieron con lo que mencionaron Zimback et al. (2011), quienes, en trabajos similares de asociación genética en especies forestales, encontraron altos valores de correlación genética entre esos caracteres a edades similares.

En el sitio 1, a edades inferiores a cuatro años la correlación de los caracteres similares entre sí y con las otras características no semejantes, mostraron en algunos casos valores bajos (inferiores a $60 \%$ ) y en algunos casos inversos (correlaciones negativas). Específicamente la altura comercial (AltCom) mostró correlaciones muy bajas con el DAP y el volumen comercial (VolCom), en los tres ensayos evaluados.

El sitio 2 a las edades de 1,5 y 4,5 años, presentaron valores de correlaciones superiores a $70 \%$ y $80 \%$, respectivamente, entre las variables DAP y volumen comercial, principalmente, lo que evidenció el potencial de selección genética a temprana edad. Los valores de correlación obtenidos fueron congruentes con el análisis de las heredabilidades, que evidenciaron la tendencia que indicó las posibilidades de selección genética entre los 2,8 y 4 años de edad. La selección temprana, al ser una selección indirecta que se puede realizar mediante correlaciones genéticas, las cuales deben ser confiables, permite su aplicación en la reproducción operativa (Rweyongeza, 2016).

Se ha demostrado que los parámetros genéticos y las correlaciones genéticas edad - edad difirieron entre sitios o regiones geográficas (Gwaze et al., 2000; Weng et al., 2007), sin embargo, a pesar de las diferencias obtenidas en los coeficientes de heredabilidad individual y heredabilidad media del clon, así como en los demás parámetros genéticos, con el análisis integrado, la tendencia en el comportamiento de la especie se mantuvo en los tres sitios, a partir del cual fue posible proyectar la edad de selección. 


\section{Conclusiones}

El diámetro a la altura del pecho (DAP) y el volumen comercial fueron los caracteres con mayor control genético, y a la vez los mayores valores promedio de heredabilidad individual y media clonal, con una tendencia a aumentar dichas heredabilidades entre los 2,8 y 4 años de edad. Lo anterior se respaldó con los valores de correlación genética, lo que permitió definir dicho rango de edad como posible edad óptima de selección genética.

Las mayores correlaciones genéticas se presentaron entre las variables DAP y volumen comercial a partir los 2,7 años de edad (>80 \%), que aumentaron con edades posteriores hasta alcanzar valores mayores a $90 \%$, lo que permitió suponer el potencial de selección temprana con base a los caracteres analizados.

\section{Referencias}

Alfaro, M. A. L., Aymerich, U. N., Blanco, L. G., Bolaños, A. L., Campos, M. A., \& Matarrita, O. R. (2013). Guía de diseño bioclimático según clasificación de zonas de vida de Holdridge [Tesis de Licenciatura, Universidad de Costa Rica]. Repositorio de la Universidad de Costa Rica. http://repositorio.sibdi.ucr.ac.cr:8080/jspui/handle/123456789/2110

Barrantes, A. R., \& Ugalde, S. A. (2019). Usos y aportes de la madera en Costa Rica: Estadísticas 2018 y precios 2019. Oficina Nacional Forestal. https:/onfcr.org/media/uploads/documents/usos-y-aportes-de-la-madera-2019.pdf

Beltrame, R., Bisognin, D. A., Mattos, B. D., Cargnelutti Filho, A., Haselein, C. R., Gatto, D. A., \& dos Santos, G. A. (2012). Desempenho silvicultural e seleção precoce de clones de híbridos de eucalipto. Pesquisa Agropecuária Brasileira, 47(6), 791-796. https://doi.org/10.1590/S0100-204X2012000600009

Chen, X., Hawkins, B., Xie, C. Y., \& Ying, C. C. (2003). Age trends in genetic parameters and early selection of lodgepole pine provenances with particular reference to the lambeth model. Forest Genetics, 10(3), 249-258. https://kf.tuzvo.sk/sites/ default/files/FG10-3_249-258.pdf

de Resende, M. D. V. (2016). Software Selegen-REML/BLUP: A useful tool for plant breeding. Crop Breeding and Applied Biotechnology, 16(4), 330-339. https://doi.org/10.1590/1984-70332016v16n4a49

de Resende, M. D. V., \& Duarte, J. B. (2007). Precisão e controle de qualidade em experimentos de avaliação de cultivares. Pesquisa Agropecuária Tropical, 37(3), 182-194.

Dean, C. A., Cotterill, P. P., \& Burdon, R. D. (2006). Early selection of Radiata Pine. I. Trends over time in additive and dominance genetic variances and covariances of growth traits. Silvae Genetica, 55(1-6), 182-191. https://doi. org/10.1515/sg-2006-0025

Diao, S., Hou, Y., Xie, Y., \& Sun, X. (2016). Age trends of genetic parameters, early selection and family by site interactions for growth traits in Larix kaempferi open-pollinated families. BMC Genetics, 17(1), 104-115. https://doi.org/10.1186/ s12863-016-0400-7

Foster, G. S., \& Shaw, D. V. (1988). Using clonal replicates to explore genetic variation in a perennial plant species. Theoretical and Applied Genetics, 76, 788-794. https://doi.org/10.1007/BF00303527

Gwaze, D. P., Bridgwater, F. E., Byram, T. D., Woolliams, J. A., \& Williams, C. G. (2000). Predicting age-age genetic correlations in tree-breeding programs: A case study of Pinus taeda L. Theoretical and Applied Genetics, 100(2), 199-206. https://doi.org/10.1007/s001220050027 
Hodge, G. R., \& Dvorak, W. S. (2004). The CAMCORE international provenance/progeny trials of Gmelina arborea: Genetic parameters and potential gain. New Forests, 28(2/3), 147-166. https://doi.org/10.1023/B:NEFO.0000040942.34566.a7

Instituto de Desarrollo Rural.(2015). Caracterización del Territorio Siquirres-Guácimo. http://obturcaribe.ucr.ac.cr/documentospublicaciones/planes-y-programas-n/inder/187-caracterizacion-siquirres-guacimo

Instituto Meteorológico Nacional. (2005). Atlas Climatológico de Costa Rica. https://www.imn.ac.cr/atlas-climatologico.

Kumar, A. (2007). Growth Performance and variability in different clones of Gmelina arborea (Roxb.). Silvae Genetica, 56(1-6), 32-36. https://doi.org/10.1515/sg-2007-0005

Lauridsen, E. B., \& Kjaer, E. D. (2002). Provenance research in Gmelina arborea Roxb. A summary of results from three decades of research and a discussion of how to use them. International Forestry Review, 4(2), 1-15.

Lokmal, N. (1994). Genetic parameters of Gmelina arborea: height and diameter growth. Journal of Tropical Forest Science, 7(2), 323-331. http://www.jstor.org/stable/43581818

Mata-Chinchilla, R., \& Castro-Chinchilla, J. (2019). Geoportal de suelos de Costa Rica como bien público al servicio del país. Revista Tecnología en Marcha, 32, 51-56. https://doi.org/10.18845/tm.v32i7.4259

Moraes, C. B., Brizolla, T. F., Teixeira, L. G., Zimback L., Tambarussi, E. V., Chaves, R., \& Mori, E. S. (2014). Estimates of genetic parameters for selection of trees in Eucalyptus. Scientia Forestalis, 42(104), 623-629. http://www.ipef.br/ publicacoes/scientia/nr104/cap16.pdf

Moya, R. (2004). Gmelina arborea en Costa Rica. Bois et Forest Des Tropiques, 279(1), 47-57.

Murillo, O., Meza, A., \& Cabrera, J. M. (2004). Estimación del valor real y del valor de mercado en pie de la plantación forestal. Revista Agronomía Costarricense, 28(1), 47-55. http://www.mag.go.cr/rev_agr/v28n01_047.pdf

Padua, F. M. (2004). Juvenile selection of Gmelina arborea clones in the Philippines. New Forests, 28(2/3), 195-200. https:// doi.org/10.1023/B:NEFO.0000040946.46722.1c

Pavlotzky, B., \& Murillo, O. (2014). Ganancia genética esperada e interacción genotipo-ambiente en Acacia mangium en la zona norte de Costa Rica. Agronomía Costarricense, 38(2), 7-17. https://doi.org/10.15517/rac.v38i2.17270

Rweyongeza, D. M. (2016). A new approach to prediction of the age-age correlation for use in tree breeding. Annals of Forest Science, 73(4), 1099-1111. https://doi.org/10.1007/s13595-016-0570-5

Santos, A. M., Rosado, S. C. da S., \& Oliveira, A. N. (2014). Estimation of genetic parameters and verification of early selection efficiency in baru (Dipteryx alata). Crop Breeding and Applied Biotechnology, 14(4), 238-243. https://doi. org/10.1590/1984-70332014v14n4a37

Weng, Y. H., Tosh, K. J., Park, Y. S., \& Fullarton, M. S. (2007). Age-related trends in genetic parameters for Jack Pine and their implications for early selection. Silvae Genetica, 56(1-6), 242-252. https://doi.org/10.1515/sg-2007-0035

Wijoyo, F. S. (2000). A study of genetic parameters of Gmelina arborea Roxb. From Thailand grown in 5 countries [Unplublished Magister Sience dissertation] North Carolina State University.

Xiang, B., Li, B., \& Isik, F. (2003). Time trend of genetic parameters in growth traits of Pinus taeda L. Silvae Genetica, 52(3-4), 114-121.

Ye, T. Z., \& Jayawickrama, K. J. S. (2012). Early selection for improving volume growth in Coastal douglas-fir breeding programs. Silvae Genetica, 61(1-6), 186-198. https://doi.org/10.1515/sg-2012-0024 
Zimback, L., Mori, E. S., Brizolla, T. F., \& Chaves, R. (2011). Correlações entre caracteres silviculturais durante o crescimento de Eucalyptus grandis Hill ex Maiden. Revista do Instituto Florestal de São Paulo, 23(1), 57-67. http://hdl.handle. net/11449/141157 